\title{
Report on Models of Write-Efficient Memories with Localized Errors and Defects
}

\author{
R. Ahlswede and M.S. Pinsker
}

\begin{abstract}
Write-efficient memories (WEM) as a model for storing and updating information were introduced by R. Ahlswede and Z. Zhang [2]. We consider now three new models of WEM with localized errors and defects, resp.

In the situation $\left(E_{+}, D_{-}\right)$, where the encoder is informed but the decoder is not informed about the previous state of the memory we study

1. WEM codes correcting defects,

2. WEM codes detecting localized errors.

Finally, in the situation $\left(E_{+}, D_{+}\right)$, where both, the encoder and the decoder, are informed about the previous state of the memory we study.

3. WEM codes correcting localized errors.

In all three cases we determine for binary alphabet the optimal rates under a cost constraint defined in terms of the Hamming distance.
\end{abstract}

\section{Introduction}

We recall first the model and a result of [2]. A write-efficient memory (WEM) is a model for storing and updating information on a rewritable medium. There is a cost $\varphi: \mathcal{X} \times \mathcal{X} \rightarrow \mathbb{R}_{\infty}$ assigned to changes of letters. A collection of subsets $\mathcal{C}=\left\{C_{i}: 1 \leq i \leq M\right\}$ of $\mathcal{X}^{n}$ is an $(n, M, d)$ WEM code, if

$$
C_{i} \cap C_{j}=\varnothing \text { for all } i \neq j
$$

and, if

$$
d_{\max }=\max _{1 \leq i, j \leq M} \max _{x^{n} \in C_{i}} \min _{y^{n} \in C_{j}} \sum_{k=1}^{n} \mathcal{C}\left(x_{k}, y_{k}\right) \leq d .
$$

$d_{\max }$ is called the minimax correction cost with respect to the given cost function $\varphi$. The performance of a code $\mathcal{C}$ can also be measured by two parameters, namely, the maximal cost per letter $\delta_{\mathcal{C}}=n^{-1} d_{\max }$ and the rate of the size $R_{\mathcal{C}}=n^{-1} \log M$. The rate achievable with a maximal per letter cost $\delta$ is thus $R(\delta)=\sup _{\mathcal{C}: \delta_{\mathcal{C}} \leq \delta} R_{\mathcal{C}}$.

This is the most basic quantity, the storage capacity of a $\operatorname{WEM}\left(\mathcal{X}^{n}, \varphi^{n}\right)_{n=1}^{\infty}$. It has been characterized in [2] for every $\varphi$.

The operational significance of a WEM code is as follows. For a set $\mathcal{M}=$ $\{1,2, \ldots, M\}$ of possible messages and the state $x^{n}=\left(x_{1}, \ldots, x_{n}\right) \in C=\bigcup_{i=1}^{M} C_{i}$ of the memory the encoder can store any message $i \in \mathcal{M}$ by any state $y^{n}$ in $C_{i}$. 
It is guaranteed that the cost of changing $x^{n}$ to $y^{n}$ does not exceed $d$, provided that the encoder knows the original state $x^{n}$. The decoder votes for message $j$, if $y^{n} \in C_{j}$. He does not use and therefore does not need knowledge about the previous state $x^{n}$. We are thus in a case $\left(E_{+}, D_{-}\right)$.

In this model there is no error in encoding and decoding. Solely the cost function $\varphi$ defines it. Clearly, by the forgoing explanation the encoder can update any message $i$ stored by a state of $C_{i}$ to any message $j$ stored by a suitable state in $C_{j}$.

In this note we confine ourselves to the binary Hamming case, that is, $\mathcal{X}=$ $\{0,1\}$ and the encoder cannot change symbols in more than $d=\delta n, 0 \leq \delta \leq \frac{1}{2}$, positions of a state. In this case the result of [2] specialized to

$$
R(\delta)=h(\delta),
$$

where $h(\delta)=-\delta \log \delta-(1-\delta) \log (1-\delta)$.

WEM codes can be considered, where we take instead of $\mathcal{X}^{n}$ a subset with restrictions on codewords, for instance on the weight of the codewords, on the distribution of 0 and 1 , etc.

Such a partition can be used in some other situations, for example WEM codes correcting defects, WEM codes detecting localized errors, i.e. the encoder has an additional information about errors.

In Section 2 we present our three models. In Section $3-5$ we present capacity theorems for them.

\section{Three Models of WEM with Errors}

We study three types of WEM codes with additional properties.

\section{WEM code correcting $t=\tau n$ defects $\left(E_{+}, D_{-}\right)$}

Denote the $t$ element subsets of $[n]=\{1,2, \ldots, n\}$ by $\mathcal{E}_{t}=\left(\begin{array}{c}{[n]} \\ t\end{array}\right)$. Any $E \in \mathcal{E}_{t}$ can be the set of defect positions and any $e_{E}=\left(e_{k}\right)_{k \in E}$ can specify the defects $e_{k}$ in position $k$. Both, $E$ and $e_{E}$, are known to the encoder, who also knows the present state $x^{n}$ whereas the decoder knows nothing.

The decoder, reading $y^{n} \in C_{j}$ votes for message $j$. Necessarily the $C_{j}$ 's are again disjoint. Moreover, for every $x^{n} \in C=\bigcup_{j=1}^{M} C_{j}, E$, and $e_{E}$ there must be for every $j$ a $y^{n} \in C_{j}$ with

$$
y_{k}=e_{k} \text { for } k \in E
$$

and

$$
\sum_{k \in[n] \backslash E} \varphi\left(d_{k}, y_{k}\right) \leq d
$$

\section{WEM code detecting $t$ localized errors $\left(E_{+}, D_{-}\right)$}

Any $E \in \mathcal{E}_{t}$ can be the set of positions with possible errors. At any updating $E$ is known to the encoder. We want to be able to have a large set $\mathcal{M}=\{1,2, \ldots, M\}$ 
of messages as candidates for an updating. The code $\left\{C_{i}: 1 \leq i \leq M\right\}$ has to be designed such that the decoder decides correctly, if no error occurs in the positions $E$, and otherwise he detects an error.

It will be shown that optimal rates are achieved with codes of the following structure:

The elements in $C=\bigcup_{i=1}^{M} C_{i}$ have weight $\left\lfloor\frac{n-t}{2}\right\rfloor$.

For $j \in \mathcal{M}$ and $x^{n} \in C$ we require that $y^{n}=y^{n}\left(x^{n}, E, j\right)$ satisfies $y_{k}=x_{k}$ for $k \in E, y^{n} \in C_{j}$, and $D\left(x^{n}, y^{n}\right) \leq d$.

If for the output state $v v \in C_{j}$, then $v$ is decoded into $j$, and if $v \notin C_{j}$, then necessarily $|v|>\left\lfloor\frac{n-t}{2}\right\rfloor$ and an error is detected.

Actually, we shall make sure by additional assumptions on the model that always the state sequence $x^{n}$ satisfies $x_{k}=0$ for $k \in E$.

This can be achieved by a.) having the same $E$ (unknown to the decoder for all updatings) or by b.) allowing only errors which change a 1 into a 0 .

In case of a detection the memory has to be cleaned by writing a 0 everywhere.

\section{WEM code correcting $t$ localized errors $\left(E_{+}, D_{+}\right)$}

Let $E \in \mathcal{E}_{t}$ be the set of possible positions for errors in updating. It is known to the encoder, who also knows the present state, say $x^{n}$. He encodes a message $j \in\{1,2, \ldots, M\}$ by a $y^{n}\left(x^{n}, j, E\right)$ satisfying $D\left(x^{n}, y^{n}\right) \leq d$. For the output state $v D\left(y^{n}, v\right) \leq t$ holds. The decoder knows $x^{n}$ and bases his decoding $\Psi$ on $x^{n}$ and $v$. Therefore $\Psi: \mathcal{X}^{n} \times \mathcal{X}^{n} \rightarrow \mathcal{M}$ must satisfy $\Psi\left(x^{n}, v\right)=j$.

Of course by the distance constraint there is no loss in assuming that $y_{k}=x_{k}$ for $k \in E$. Since both, encoder and decoder, know $x^{n}$ the coding problem is practically equivalent to the coding problem for transmission in the presence of localized errors, except that there is now the constraint for the codewords to have weights not exceeding $d$.

Remark 1. An interesting more difficult coding problem arises, if model 3 is altered such that the encoder does not remember the previous state, that is, we are in case $\left(E_{+}, D_{-}\right)$.

\section{The Capacity in Model 1}

We denote by $R_{\text {def }}(\delta, \tau)$ the optimal rate, that is, the capacity of WEM codes correcting $t=\tau n$ defects at maximal cost $d=\delta n$. We need the quantity

$$
R(w, \tau)= \begin{cases}(1-\tau) h\left(\frac{w}{1-\tau}\right), & \text { if } w \leq \frac{1}{2}(1-\tau) \\ 1-\tau, & \text { if } w \geq \frac{1}{2}(1-\tau) .\end{cases}
$$

Theorem 1. For any $0 \leq \tau \leq 1$ and $0 \leq \delta \leq 1$

$$
R_{\text {def }}(\delta, \tau)=R(\delta, \tau) .
$$


We recall the Color Carrying Lemma. (see [2])

The hypergraph $\mathcal{H}=(\mathcal{V}, \mathcal{E})$ carries $M$ colors, if $M \leq\left(\ell n|\mathcal{E}| \min _{E \in \mathcal{E}}|E|\right)^{-1} \min _{E \in \mathcal{E}}|E|$.

Remark 2: Misprints in Lemma in [2] have been corrected.

\section{Proof of Theorem 1}

For $x^{n} \in \mathcal{X}^{n}$ and $x_{k}=e_{k}(k \in E)$ define

$$
\mathcal{S}_{d}\left(x^{n}, E, e_{E}\right)=\left\{y^{n} \in \mathcal{X}^{n}: y_{k}=e_{k} \text { for } k \in E, D\left(y^{n}, x^{n}\right) \leq d\right\} .
$$

Clearly, $M \leq\left|\mathcal{S}_{d}\left(x^{n}, E, e_{E}\right)\right|$ and $\left|\mathcal{S}_{d}\left(x^{n}, E, e_{E}\right)\right|=\sum_{i=0}^{d}\left(\begin{array}{c}n-t \\ i\end{array}\right) \sim \exp \{R(\delta, \tau) n\}$.

By applying the previous lemma to the hypergraph with vertex set $\mathcal{X}^{n}$ and edges defined in (6) we can achieve the rate $R(\delta, \tau)$.

Remark 3: Actually, Theorem 1 is also an immediate consequence of the results of $[3]$.

\section{The capacity for model 2}

Here $R_{\text {loc }}^{\text {dect }}(\delta, \tau)$ denotes the optimal rate (capacity) of WEM codes detecting $t=\tau n, 0 \leq \tau \leq \frac{1}{2}$, localized errors with cost $d=\delta n$. The encoder knows the set $E$ of error positions $E$ and the previous state of the memory.

Theorem 2. Under conditions a.) or b.)

$$
R_{\mathrm{loc}}^{\mathrm{detect}}(\delta, \tau)=R(\delta, \tau) .
$$

\section{Remarks}

4. The expression $R(\delta, \tau)$ also occurs as the capacity in correcting localized errors with constant weight $\delta n$ codes (see [4]). That result also follows from the proof of Theorem 2 .

5. The work of [6] does not assume restrictions on the weight of codewords.

Proof: The inequality $R_{\mathrm{loc}}^{\text {detect }}(\delta, \tau) \leq R(\delta, \tau)$ is obvious and the opposite inequality follows again by the Color Carrying Lemma.

\section{The capacity for model 3}

We denote by $R_{\text {loc }}(\delta, \tau)$ the optimal rate (capacity) of WEM codes correcting $t=\tau n, 0<\tau \leq \frac{1}{2}$, localized errors with cost $d=\delta n$. Recall that the encoder knows the set of error positions $E$ and both encoder and decoder, are informed about the previous state of the memory.

We define now a quantity $R^{L}$ which describes the capacity in correcting $t=\tau n$ localized errors with binary constant weight $w=w n, 0<w \leq 1$, codes

$$
R^{L}(w, \tau)= \begin{cases}h(w+\tau)-h(\tau), & \text { if } 0<w \leq \frac{1}{2} \\ 1-h(\tau), & \text { if } \frac{1}{2}-\tau \leq w \leq \frac{1}{2} \\ h(w-\tau)-h(\tau), & \text { if } \frac{1}{2}+\tau \leq w<1\end{cases}
$$

(see [4]). 
Combining this result with the capacity theorem of [2] for error free WEM codes one gets

\section{Theorem 3}

$$
R_{\mathrm{loc}}(\delta, \tau)=R^{L}(\delta, \tau)
$$

Remark 6. Some facts about WEM codes in the situation $\left(E_{+}, D_{+}\right)$can be carried over to ordinary codes with constant weight.

Theorem 3 can be extended to the situation $\left(E_{+}, D_{-}\right)$, when positions $E$ of $t$ possible errors do not change during updating, namely, we have the capacity $R_{\mathrm{loc}}^{0}(\delta, \tau)$.

\section{Theorem 4}

$$
R^{L}(w, \tau) \leq R_{l o c}^{0}(\delta, \tau) \leq R^{L}(\delta, \tau),
$$

where $w$ is defined by the equation

$$
\delta=2 w(1-w) .
$$

However, if $w+\tau \geq \frac{1}{2}$, then we have equalities in (8).

\section{References}

1. R. Ahlswede and M.S. Pinsker, Report on models of write-efficient memories with localized errors and defects, Preprint 97-004 (Ergänzungsreihe), SFB 343 "Diskrete Strukturen in der Mathematik", Universität Bielefeld, 1997.

2. R. Ahlswede and Z. Zhang, Coding for write efficient memories, Inform. and Computation, Vol. 83, No. 1, 80-97, 1989.

3. R. Ahlswede, L.A. Bassalygo, and M.S. Pinsker, Binary constant weight codes correcting localized errors and defects, Probl. Inform. Trans., Vol. 30, No. 2, 10-13, 1994.

4. L.A. Bassalygo and M.S. Pinsker, Binary constant-weight codes correcting localized errors, Probl. Inform. Trans., Vol. 28, No. 4, 103-105, 1992.

5. A.V. Kusnetsov and B.S. Tsybakov, Coding a memory with defective cells, Probl. Inform. Trans., Vol. 10, No. 2, 52-60, 1974.

6. L.A. Bassalygo and M.S. Pinsker, Codes detecting localized errors, preprint. 